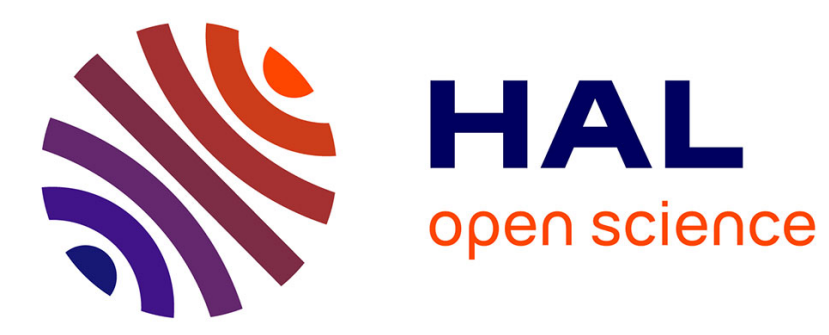

\title{
Chemical conjugation of nucleic acid aptamers and synthetic polymers
}

Maria Nerantzaki, Capucine Loth, Jean-Francois Lutz

\section{To cite this version:}

Maria Nerantzaki, Capucine Loth, Jean-Francois Lutz. Chemical conjugation of nucleic acid aptamers and synthetic polymers. Polymer Chemistry, 2021, 12 (24), pp.3498-3509. 10.1039/D1PY00516B . hal-03454585

\section{HAL Id: hal-03454585 \\ https://hal.science/hal-03454585}

Submitted on 29 Nov 2021

HAL is a multi-disciplinary open access archive for the deposit and dissemination of scientific research documents, whether they are published or not. The documents may come from teaching and research institutions in France or abroad, or from public or private research centers.
L'archive ouverte pluridisciplinaire $\mathbf{H A L}$, est destinée au dépôt et à la diffusion de documents scientifiques de niveau recherche, publiés ou non, émanant des établissements d'enseignement et de recherche français ou étrangers, des laboratoires publics ou privés. 


\title{
Chemical conjugation of nucleic acid aptamers and synthetic polymers
}

\author{
Maria Nerantzaki, ${ }^{*}$ Capucine Loth and Jean-François Lutz* \\ Université de Strasbourg, CNRS, Institut Charles Sadron UPR22, 23 rue du Loess, 67034 \\ Strasbourg Cedex 2, France. \\ Maria Nerantzaki : nerantzaki@unistra.fr \\ Jean-François Lutz: jflutz@unistra.fr
}

Published in Polymer Chemistry, 12, 3498-3509 (2021)

https://pubs.rsc.org/en/content/articlelanding/2021/py/d1py00516b 


\begin{abstract}
Nucleic acid aptamers are chemically-synthesized single-stranded oligonucleotides that fold into specific sequence-dependent configurations. Due to their exceptional recognition properties towards a variety of biological targets, they find applications in many areas of bioscience such as affinity chromatography, drug delivery, biosensors, diagnostics, stem cell research and regenerative medicine. In recent years, aptamers conjugation to synthetic polymers has gained increasing attention, as an effective strategy to control biochemical parameters, uptake mechanisms and pharmacokinetic properties. In this review, we summarize and critically discuss the different approaches that have been used so far for the preparation of aptamer-polymer conjugates. Most of the reported work has been achieved by reacting an aptamer with a functional polymer in solution. Different ligation chemistries such as active ester amidation, thio-Michael addition, direct thiol-disulfide exchange and azide-alkyne Huisgen cycloaddition have been used for that purpose. Alternatively, polymer conjugation can be performed on solid-supports. After chemical synthesis, resin bound aptamers are reacted with a macromolecular reagent or extended by phosphoramidite polymer chemistry. Furthermore, some non-covalent strategies, such as polyelectrolyte complexation, have been reported for the preparation of aptamer-polymer conjugates. The pro and cons of all these strategies are discussed herein.
\end{abstract}




\section{Introduction}

Aptamers (from the Greek word $\ddot{\alpha} \pi \tau \omega$, which means "to fasten to") is a general term for short-chain nucleic acids (also sometimes, but more rarely, peptides) that recognize specific molecules. ${ }^{1,2}$ Due to their outstanding recognition capability, numerous nucleic acid aptamers (DNA or RNA) have been screened and used in major bio-applications including drug delivery, diagnostics, bio-separation and theranostics. ${ }^{3-6}$ Yet, in all these applications, aptamers are rarely used as such and are usually connected to other components, which can be other biomolecules, drugs, fluorescent markers, inorganic surfaces and synthetic polymers. The latter aspect is particularly important since it is well-known that the attachment of synthetic macromolecules to biological segments (a process known as polymer bioconjugation) allows unique combinations of man-made and natural properties. ${ }^{7,8}$ For instance, polymer bioconjugation permits to overcome inherent disadvantages of nucleic acid aptamers in biomedical applications, such as instability against enzymatic degradation and rapid renal clearance. This can, for example, be achieved using poly(ethylene glycol) (i.e. PEGylation), ${ }^{9}$ which has been shown to improve significantly the pharmacokinetics of therapeutic aptamers. In fact, PEGylation allowed the synthesis of the first, and so far only, FDA-approved aptamerbased drug known as PEGaptanib (Macugen $\left.{ }^{\circledR}\right) .{ }^{10}$ In this formulation, a 27-mer RNA aptamer, which binds the vascular endothelial growth factor (VEGF)-165, is modified with a branched PEG structure at its 5'-terminus. Conjugation with high molecular weight PEG reduces renal filtration rate, improves aptamer retention in vivo and consequently extends aptamer half-life up to 48 hours.

Over the past years, the success story of Macugen has triggered further research about the synthesis of aptamer-polymer conjugates (APCs). Several types of synthetic macromolecules, including biodegradable polymers, amphiphilic block copolymers, 
graft copolymers, conducting polymers and dendrimers have been investigated for aptamers conjugation. Still, the motivations behind these polymer conjugations can be quite diverse and triggered by different applications. For example, amphiphilic APCs, which self-assemble into stable nanostructures in aqueous medium, are mainly studied as drug delivery vectors and have been shown to combine improved nuclease resistance and enhanced cellular penetration. ${ }^{11}$ On the other hand, aptamer-embedded hydrogels have been principally explored in bio-assays and have been tested as signalling complexes, which could broaden traditional fluorescence-based assays to visual detection. ${ }^{12}$ Several excellent recent reviews discuss in detail the properties and potential applications of aptamer-based materials. ${ }^{6,13-15}$ However, although a large number of APCs have been described in the literature, the present review provides for the first time an overview of the synthetic pathways that are used for their synthesis. It shall be clarified that the content of this review is intentionally restricted to nucleic acid-based APCs and does not include conjugation works that have been done with peptide aptamers.

Overall, the content of this review is of interest from both a chemistry and biomaterials perspective, as it sheds light on functional APCs and their use, but also from a synthetic chemistry angle, as the preparation of this bio-hybrid macromolecules requires efficient synthetic tools that works at the interface between polymer and nucleic acid chemistry. Still, this text is primarily written for chemists and polymer scientists and therefore in-depth information about the biological properties of aptamers is intentionally not provided. Yet, for the reader's convenience, Figure 1 and Table 1 depict the aptamers that are discussed in this review. In terms of synthetic strategy, most of the works listed in this review rely on the covalent ligation of macromolecular reagents, which can be performed in solution and or on a solid-support. Still, some other strategies 
have been explored such as controlled polymer growth from an aptamer macroinitiator. Additionally, some APCs have been prepared using supramolecular strategies such as polyelectrolytes complexation. Consequently, we have classified here the different synthetic strategies based on the use of covalent or non-covalent interactions. More precisely, the covalent strategies have been subdivided into solution and solid-phase approaches, while electrostatic interactions, are discussed as an alternative non-covalent approach.

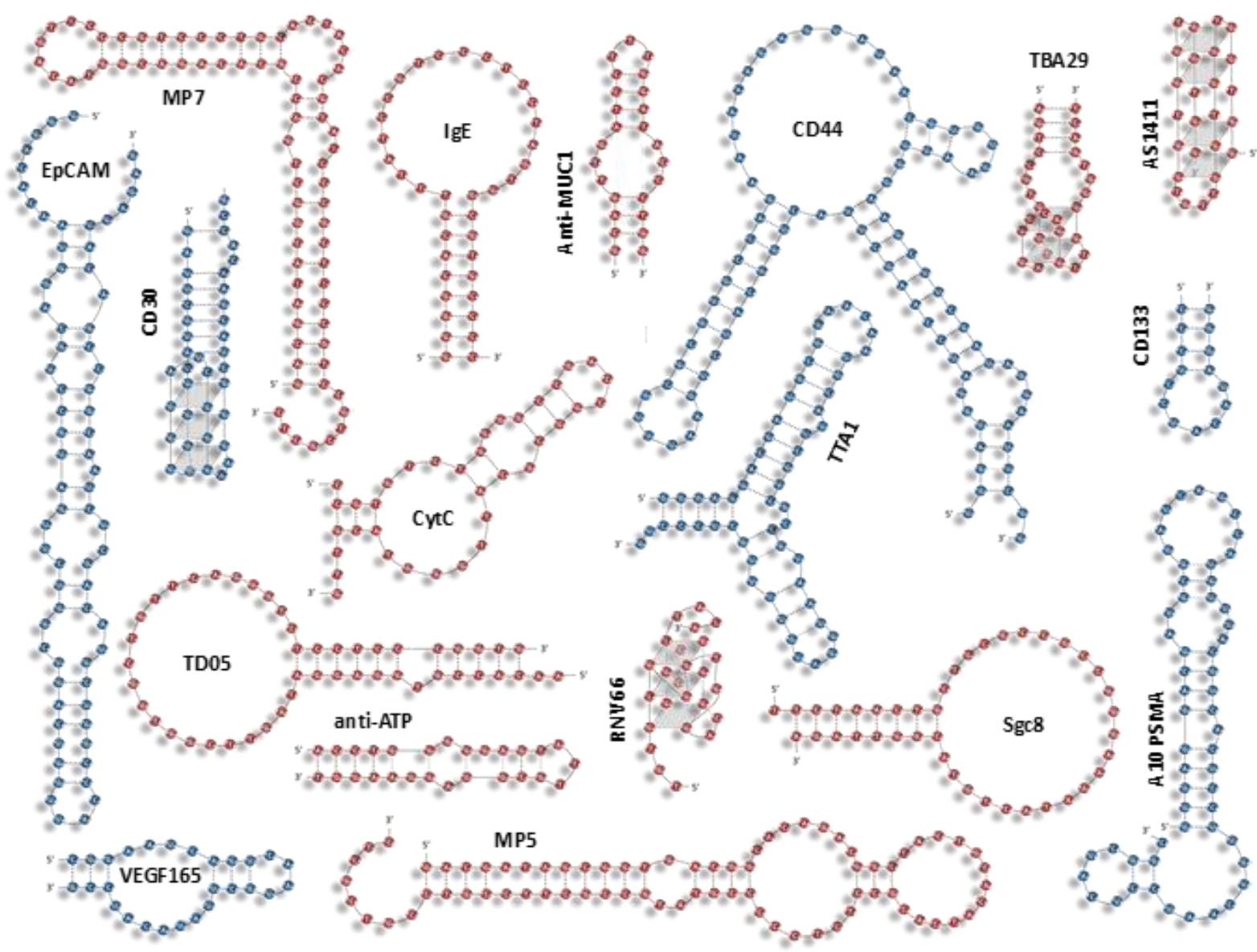

Figure 1. Schematized structure of all the aptamers that are discussed in this review. Structures drawn in red and blue represent DNA and RNA aptamers, respectively. The letters A, T, G, C and U denotes classical DNA or RNA nucleotides. 
Table 1. Some classical nucleic acid aptamers.

\begin{tabular}{llll}
\hline Name & Type $^{\mathbf{a}}$ & Target & Ref \\
\hline TBA & 15-mer DNA & Thrombin protein & 16 \\
RNV66 & 25-mer DNA & Vascular endothelial growth factor & 17 \\
anti-MUC1 & 25-mer DNA & MUC1 protein & 18 \\
AS1411 & 26-mer DNA & Nucleolin protein & 19 \\
anti-ATP & 27-mer DNA & ATP, AMP, adenosine & 20 \\
IgE & 37-mer DNA & Immunoglobulin E & 21 \\
CytC & 40-mer DNA & Cytochrome-C & 22 \\
Sgc8 & 41-mer DNA & Tyrosine kinase 7 & 23 \\
MP-5 & 61-mer DNA & Murine PD-1 & 24 \\
MP-7 & 75-mer DNA & Murine PD-1 & 24 \\
CD30 & 39-mer RNA & CD30 protein & 26 \\
anti-PSMA & 43-mer RNA & prostate-specific membrane antigen & 26 \\
TTA1 & 55-mer RNA & Tenascin-C protein & 24 \\
EpCAM & 73-mer RNA & Epithelial cell adhesion molecule & 26 \\
\hline & & & 24 \\
\hline
\end{tabular}

${ }^{a}$ The chain-lengths indicated in this table correspond to standard examples. The sequence of a given aptamer may be elongated or truncated. For instance, the aptamers discussed in this review or shown in Figure 1 are sometimes longer or shorter derivatives of those listed here.

\section{Covalent conjugation}

Among all the strategies available for bioconjugation, covalent conjugation, which involves the formation of a covalent bond between the polymer and the aptamer, is the most widely used method for the preparation of APCs. As described in a classical review about polymer 
bioconjugates, ${ }^{7}$ four different approaches can be utilized to form a covalent bond between a biomacromolecule and a synthetic polymer: (i) coupling/ligation, (ii) macroinitiators, (iii) inverse bioconjugation and (iv) macromonomers. Most of the examples discussed in this section were obtained using the first approach (Figure 2) and to a lower extent using the second and fourth ones. These strategies can be performed in solution or on a solid support. In the former case, all reactants are solubilized in a common solvent, whereas in the latter, one reactive segment (typically the aptamer) is immobilized on a crosslinked solid. To select the most suitable method, many factors including structure, stability and solubility of terminal moieties have to be considered. In general, solution ligation shows great advantage for aptamers conjugation to hydrophilic polymers. On the contrary, solid-phase functionalization gives access to a wider range of solvents and to easier purifications. In the next paragraphs, solutionphase conjugation strategies, are first elaborated upon, followed by solid-phase conjugation procedures.

\subsection{Solution strategies}

\subsubsection{Active ester amidation}

The formation of an amide linkage, based on the condensation of activated carboxylic acids and amines, is one of the most frequently used coupling strategy for the conjugation of synthetic polymers and biomolecules (Figure $2 a){ }^{7}$ In particular, activation of carboxyl groups of polymers using 1-ethyl-3-(3-dimethylaminopropyl) carbodiimide (EDC) in the presence of $N$-hydroxy-succinimide (NHS), and coupling to an amine-modified aptamer, has been proven to be particularly advantageous for the synthesis of various aptamer-polymer conjugates, for application in cancer diagnosis and therapy. Hicke and coworkers reported one of the earliest examples of aptamer PEGylation using this convenient chemistry. ${ }^{29}$ They modified the TTA1 aptamer, which 
targets the extracellular matrix protein tenascin-C, by conjugating the $5^{\prime}$-amino modified aptamer to a PEG chain containing an NHS-activated moiety and a terminal technetiumbinding ligand. In a more recent study, ${ }^{30}$ Neoh and coworkers used the NHS/EDC coupling method for PEGylation of the anti-MUC1 aptamer, which has been shown to be an efficient bioreceptor for the breast cancer biomarker mucin 1 (MUC1) protein. ${ }^{31}$ Using agarose gel electrophoresis to determine the extent of reaction and keeping EDC/NHS in excess, several parameters that may affect PEGylation of aptamer, including PEG to aptamer molar ratio, ionic strength and reaction time were investigated. The aptamer/PEG ratio for optimal conjugation was found to be 1:60, while ionic strength and reaction time did not significantly influence the PEGylation degree of the aptamer. The aptamer-polymer conjugate was loaded with the drug doxorubicin. The incubation of MUC1 positive breast cancer cells (MC7) with the resulting conjugates confirmed that PEGylation increased the targeting efficacy of MUC1-targeting aptamer, by reducing the non-specific uptake of doxorubicin into tumor cells. ${ }^{30}$ Following a similar strategy, Gariépy and coworkers have recently described the PEGylation of hexylamine-modified aptamers MP5 and MP7, which antagonize the programmed celldeath protein $\mathrm{PD}-1 .^{24}$ The aptamers were modified at their 5' termini with a $40 \mathrm{kDa}$ mPEG-succinimidyl glutarate ester. After purification by reverse-phase HPLC, the in vivo antitumor protective properties of the PEGylated anti-PD-1 aptamers were studied. The obtained results revealed that PEGylation improves the pharmacokinetic properties of the aptamers. ${ }^{24}$

Besides conventional PEGylation, a number of research groups have explored NHSbased amidation to attach DNA to polymeric nanoparticles. For instance, poly (lactideco-glycolide) (PLGA) nanoparticles have been extensively studied, since their clinical safety is well established. ${ }^{11,32,33}$ The first example of aptamer-functionalization of 
PLGA nanoparticles was reported by Langer and coworkers. ${ }^{32}$ In this work, PLGA- $b$ PEG copolymers containing terminal carboxylate groups were used for the preparation of docetaxel-encapsulated nanoparticles by a simple nanoprecipitation method. Then, using the EDC and NHS as coupling agents, the terminal acid groups of the PEG corona of the nanoparticles were coupled to 5'-amino groups of the A10 2-fluoropyrimidine RNA aptamers that recognizes the extracellular domain of the prostate-specific membrane antigen (PSMA). The aptamer-functionalization resulted in a 15-nm increase in nanoparticle size, thus suggesting efficient bioconjugation. A few years later, the same group reported the synthesis of Pt(IV)-encapsulated PLGA- $b$-PEG nanoparticles. ${ }^{33}$ In this study, agarose gel electrophoresis confirmed that the A10 RNA aptamer, which targets PSMA cells, was successfully conjugated on the surface of the PLGA- $b$-PEG nanoparticles using the EDC/NHS technique. In another study, ${ }^{34}$ amine-terminated A10 RNA aptamers were covalently conjugated to carboxyl functionalized lipid-PEG conjugates, which were subsequently used in the self-assembly of lipid-polymer nanoparticles. The resulting hybrid nanoparticles were comprised of a hydrophobic PLGA, a hydrophilic PEG shell, and a lipid (lecithin) monolayer at the interface of the hydrophobic core and the hydrophilic shell. These hybrid nanoparticles were found to be promising drug delivery vehicles, providing tunable size and surface charge, high drug loading yield, sustained drug release profile, favourable stability in serum, good cellular targeting ability and simple synthesis process. Following these pioneer works of the Langer group, EDC-mediated amidation has been used in several other studies to surface-decorate PLGA- and PLGA- $b$-PEG-based nanoparticles prepared by the single/multiple emulsion-solvent evaporation method. ${ }^{35-43}$ These nanoparticles have a hydrophobic core, which can be utilized for the delivery of poorly soluble molecules, and a surface hydrophilic layer which enables excellent aqueous solubility while 
providing a moiety for further conjugation to ligands, including aptamers. Recently, a PLGA-based layer-by-layer multifunctional aptamer-drug carrier system was developed by Danquah and coworkers using a double emulsion technique. ${ }^{44}$ This system was composed of three layers: a drug-loaded PLGA core, an intermediate PLGA layer coupled with thrombin-targeting DNA aptamer via EDC/NHS chemistry and a final outermost layer formulated by intercalation of poly(ethylene imine) (PEI) polymeric particles within the aptamer layer via strong electrostatic forces between electronegative aptamer molecules and cationic PEI particles.

Apart from widespread PLGA, other types of biodegradable aliphatic polyesters have been coupled to aptamers. Polylactide (PLA) homopolymer is another biodegradable and non-cytotoxic synthetic polymer that has been used extensively in the formulation of particles for biotechnology and drug delivery applications. Cheng and coworkers have investigated the synthesis of paclitaxel-loaded PLA nanoparticles by Zinc-mediated polymerization of racemic D,L-lactide, followed by nanoprecipitation. ${ }^{45}$ The formed nanoparticles were coated with A10 aptamer through EDC/NHS coupling and were shown to effectively target prostate-specific membrane antigen in a cell-specific manner. In another study, a biodegradable triblock copolymer poly(oligoethylene glycol methacrylate)-poly(caprolactone)-poly(oligoethylene glycol methacrylate) was synthesized by atom transfer radical polymerization and connected to doxorubicin via an acid-labile hydrazone bond. ${ }^{46}$ Then, nanoparticles of the functionalized triblock copolymer were prepared using the nanoprecipitation method and finally, dual targeting was achieved by attaching both folic acid and the AS1411 aptamer to the particle surfaces through EDC-NHS coupling. Other polymers than polyester have been conjugated to aptamers using comparable strategies. For instance, Luo and co-workers 
have prepared paclitaxel-loaded poly (L-c-glutamyl-glutamine) nanoparticles, which were functionalized with the aptamer AS1411 via EDC/sulfo-NHS coupling reaction. ${ }^{47}$ Amphiphilic block copolymers can also self-assemble to form defined micellar aggregates such as spherical micelles and polymersomes. Such aggregates have also been conjugated to nucleic acid aptamers using NHS chemistry. For example, Qiu and coworkers have reported the conjugation of an amino-terminated nucleolin targeting AS1411 aptamer to an amphiphilic triblock copolymer poly(ethylene oxide) poly(propylene oxide)-poly(ethylene oxide) (PEO-PPO-PEO) bearing terminal carboxylic acid groups. ${ }^{48}$ Several reactions were conducted with and without EDC and with different polymer to aptamer molar ratios. In the absence of EDC, the aptamer could not be linked to the polymer, whereas in its presence a polymer/aptamer ratio of about 5 was found to be optimal for conjugation. After coupling, the APC was used in combination with beta-cyclodextrin-linked poly(ethylene glycol)-b-polylactide block copolymers for the development of doxorubicin-loaded tumor targeting micelles using the co-solvent evaporation method. ${ }^{48}$ More recently, the EDC/NHS method has been explored for the synthesis of other AS1411 aptamer-decorated micelles prepared from various copolymers such as $\mathrm{D}$ - $\alpha$-tocopheryl polyethylene glycol- $b$-poly-( $\beta$-amino ester), ${ }^{49}$ chitosan-graft-polyethylenimine ${ }^{50}$ and PEG- $b$-polycaprolactone. ${ }^{51}$ Interesting examples of aptamer modification of polymeric vesicles were also reported. For instance, Abnous, Hadizadeh and coworkers reported the aptamer functionalization of PEG- $b$-PLGA polymersomes loaded with the anticancer agents doxorubicin and gemcitabine. ${ }^{52}$ The non-immunogenic RNA aptamer anti-EpCAM and the DNA aptamer AS1411 were connected to these polymersomes using EDC/NHS chemistry. More recently, the amine-modified AS1411 aptamer was attached to the carboxyl groups of PEG on the surface of PEG- $b$-PLA polymersomes. ${ }^{53}$ In that work, the vesicles were first 
prepared using the single emulsion method and were then covalently conjugated to the aptamer using the NHS/EDC chemistry.

Some dendritic structures were also linked to aptamers using activated esters. An early example of that was reported by Wang and coworkers. ${ }^{54}$ In this work, an antibodylike nanostructure was prepared by conjugating two IgM-specific aptamers to the surface of a G-5 poly(amido amine) (PAMAM) dendrimer containing 128 carboxyl groups. In order to control the binding distance between the two aptamers, a monovalent aptamerdendrimer conjugate was first synthesized with a standard EDC coupling method and afterwards, the bivalent antibody-like nanostructure was developed by using intermolecular hybridization. The results showed that the bivalent antibody-like nanostructure could not only bind strongly to its target cells, but it also provided a tunable binding functionality in response to a temperature shift in physiological conditions. In a more recent study published by Abnous, Ramezani and coworkers, ${ }^{55}$ the AS1411 aptamer was also coupled to a PAMAM dendrimer. Covalent conjugation of amine terminated AS1411 aptamer to modified PAMAM was performed by activated ester chemistry, while non covalent conjugation of negatively charged aptamer to modified PAMAM was obtained by electrostatic complexation ( $c f$. specific section on non-covalent conjugation). The authors concluded that both aptamer conjugations were stable and could efficiently target nucleolin positive cells (A549 cells). Mukhtar, Gong and coworkers used EDS/NHS chemistry to conjugate the A10 aptamer onto the surface of hyperbranched polymer micelles consisting of a dendritic Boltorn $\mathrm{H} 40$ core, a hydrophobic PLA inner shell, and a hydrophilic PEG outer shell. ${ }^{56}$ To achieve this, an amine-terminated A10 aptamer was solubilized in a mixture of formamide/acetonitrile and then reacted with the copolymer. To ensure that at least one A10 aptamer was 
conjugated onto each unimolecular micelle the molar ratio of aptamer/polymer was carefully optimized.

a.

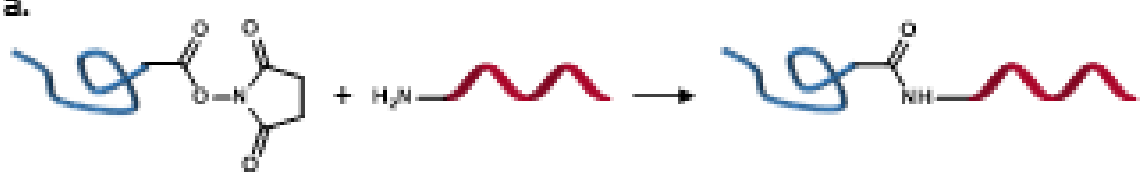

$\mathrm{O}$

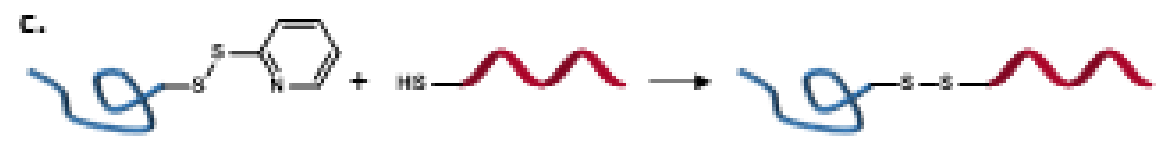

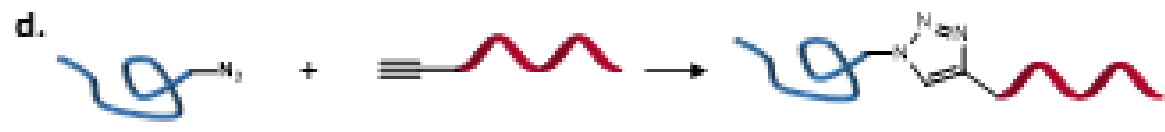

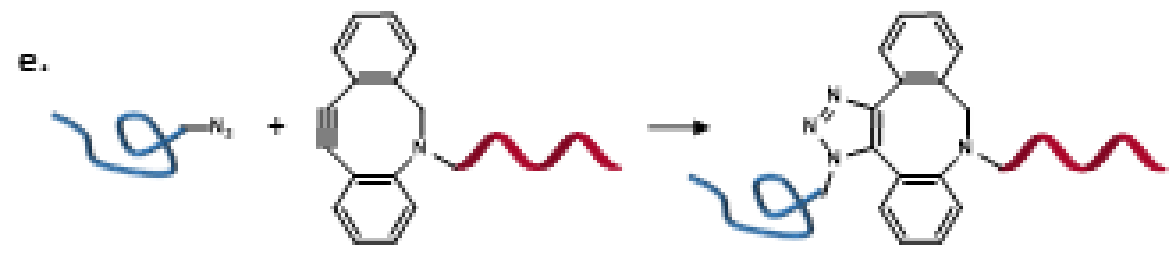

Figure 2. Classical conjugation chemistries that have been used for the preparation of APC in solution: (a) Active ester amidation; (b) Pyridyl disulfide-based thiol-disulfide exchange reaction; (c) Thiol-Michael addition; (d) Copper-catalyzed azide-alkyne cycloaddition, (e) Strain-promoted azide-alkyne cycloaddition. For clarity, additional reagents, catalysts and byproducts are intentionally not shown in this figure.

\subsubsection{Thiol-based conjugation}

Thiols are versatile reactive groups for bioconjugation chemistry. ${ }^{7}$ Indeed, these groups can be involved in a variety of reactions including thiol-ene chemistry, ${ }^{57}$ thiol-yne 
chemistry $^{58}$ and Michael-type addition of thiols to maleimides. ${ }^{59}$ In the latter case, although maleimides may hydrolyze in aqueous conditions, they react readily with thiolmodified aptamers to form a stable thioether linkage (Figure 2b). Taking advantage of this chemistry, Da Pieve, Missailidis and coworkers reported an interesting method for the PEGylation of aptamers. ${ }^{60}$ In this work, they reacted a thiol-modified anti-MUC1 aptamer with different types of maleimide-functionalized PEGs, including conventional linear PEGs, branched PEGSs and "PEG-like" poly(oligoethylene glycol) methacrylates prepared by reversible-deactivation radical polymerization. ${ }^{61}$ The aptamer initially contained disulfide protecting groups, which were reduced to thiols prior to conjugation. Afterwards, the thiol-maleimide reaction was effectively carried out under a variety of conditions. Analysis by anion-exchange HPLC analyses showed that the best conjugation yields (70-80\%) were achieved when the reaction was performed at $37{ }^{\circ} \mathrm{C}$ for $1 \mathrm{~h}$ using an aptamer/PEG molar ratio of 1:5. In a following work from the same authors, ${ }^{62}$ an anti-MUC1 aptamer containing both a primary amine and a protecting thiol was first reacted with the NHS-activated technetium chelator MAG2. After HPLC purification, the obtained product was PEGylated via the maleimide-thiol reaction, as described above. More recently, Huang, Leng and coworkers explored the same strategy to conjugate aptamers to drug-loaded micelles with a PEG corona. ${ }^{63}$ First, gefinitibloaded nanomicelles composed of PEG-distearoyl phosphatidylethanol-amine and 1,2distearoyl-sn-glycero-3-phosphoethanolamine-N-maleimide-PEG, were prepared via a lipid film-based approach. Afterwards, two cancer-targeting aptamers (CD133 and CD44) bearing a sulfhydryl group at the 5'-end, were covalently attached to the surface of the micelles by thiol-Michael addition.

Thiol-maleimide chemistry was also used for the development of aptamer-targeted dendritic drug delivery systems. For instance, Abnous, Ramezani and coworkers 
reported the conjugation of a thiol-functionalized AS1411 aptamer to the maleimide groups of PEGylated camptothecin-loaded PAMAM dendrimers. ${ }^{64}$ Successful aptamer/dendrimer conjugation was confirmed by investigating the surface charge of the dendrimers by dynamic light scattering. A comparable study was also reported by Nabavizadeh and coworkers. ${ }^{65}$ In that case, conjugation was confirmed by Fourier transform infrared spectroscopy. Guo and coworkers utilized thiol-maleimide chemistry for the aptamer modification of poly(L-lysine) dendritic structures. ${ }^{66}$ The surface groups of the dendrimers were modified with PEG and targeting ligands to achieve long circulation and mitochondria-targeting properties. To do so a PEG derivative containing a NHS-activated ester and a maleimide was used. First, the primary amine groups of the dendrimer corona were specifically reacted with the terminal NHS groups of the PEG derivative. Afterwards, the maleimide moieties of the resulting PEGylated dendrimer were reacted with the thiol groups of two thiol-terminated aptamers (AS1411 and Cyt c), thus yielding dendrimer-PEG-aptamer vectors.

Furthermore, various types of polymer nanoparticles and materials were connected to aptamers using thiol-based chemistry. For instance, aptamer-targeted PLGA nanoparticles with a polydopamine (PDA) coating have been recently prepared. ${ }^{67}$ PDA is a biocompatible biopolymer derived from self-polymerization of dopamine in alkaline aqueous solutions. ${ }^{68}$ Interestingly, PDA contains functional catechol groups, which allow it to conjugate with biomolecules containing thiol groups via Michael-type addition. In addition, compared to the traditional aptamer conjugation processes based on active groups which are prone to hydrolysis, such as NHS and maleimide, the PDAbased modification is mild and can be performed directly in an aqueous solution, thus the complexity and inefficiency of the traditional modification processes can be avoided. Considering these facts, $\mathrm{Xu}, \mathrm{Yu}$, Zhang and coworkers have synthesized and 
characterized, doxorubicin-loaded PLGA-PEG nanoparticles coated with PDA. ${ }^{67}$ These particles were surface-decorated with a 3'-functional AS1411 aptamer via a Michael addition reaction in a weak alkaline solution ( $\mathrm{pH}$ 8.0). Dynamic light scattering studies confirmed successful surface modification and aptamer conjugation. Similarly, PDAcoated nanoparticles based on a random star-shaped copolymer of caprolactone and lactic acid were covalently modified with the thiol-functional AS1411 aptamer. ${ }^{69}$ In this case, conjugation was assessed by Fourier-transform infrared spectroscopy and X-ray photoelectron spectroscopy. In the same context, molecular recognition gating membranes were developed by Sugawara, Tamaki and Yamaghchi using an aptamer as a receptor. ${ }^{70}$ Using disulfide conjugation, a thiol-modified thrombin-binding aptamer was conjugated onto grafted polymer membranes prepared from $\operatorname{poly}(N-$ isopropylacrylamide), acrylamide and a polymerizable pyridyl disulfide monomer. In this case, the conjugation was performed through a reaction between the terminal thiol group of the aptamer and the pyridyl disulfide of the grafted polymer (Figure 2c).

\subsubsection{Azide-alkyne cycloaddition reactions}

Azide-alkyne Huisgen cycloaddition is a reaction that has been widely explored in polymer chemistry, materials science and biosciences. ${ }^{71,72}$ Although metal-free or Ag-, $\mathrm{Ru}-$, Ir- and Ni-catalyzed cycloadditions of azides and alkynes have been reported, the copper-catalyzed azide-alkyne cycloaddition (CuAAC) is undoubtedly the most widelyused version of this popular reaction (Figure 2d). Yet, although CuAAC has been widely used for the synthesis of polymer bioconjugates, copper-catalysis may damage biomolecules with sensitive functional groups such as antibodies or aptamers. ${ }^{72}$ To overcome this limitation, Hawker, Soh and coworkers investigated a ligand-accelerated CuAAC reaction for coupling DNA aptamers to synthetic polymers (Figure 3 ). ${ }^{73}$ This 
strategy relies on the use of a tricarboxylate ligand, which stabilizes $\mathrm{Cu}$ (I) during the CuAAC and thus, greatly minimizes DNA damage. In particular, this ligand allowed the efficient and site-specific conjugation of an azido-functional AS1411 aptamer with an $\omega$-alkyne-functionalized polyether block copolymer, composed of ethylene glycol and ethylene glycol vinyl glycidyl ether blocks. The resulting aptamer-polymer conjugates were used as doxorubicin drug-carriers. Interestingly, they were found to be actively internalized via endocytosis into MCF-7 cells and selectively killed nucleolin-expressing target cells. Alternatively, metal-free cycloadditions were also investigated to avoid nucleic damages in aptamer-polymer conjugation. Initially reported by Bertozzi and coworkers, ${ }^{74}$ strain-promoted azide-alkyne cycloaddition (SPAAC) has been shown to be a very convenient reaction for chemical modification of sensitive biomolecules (Figure 2e). ${ }^{75}$ For instance, dibenzocyclooctyl (DCBO) derivatives can be reacted with azides via metal-free cycloaddition in organic solvents, for example in dimethyl sulfoxide (DMSO) or in aqueous buffers. SPAAC has been recently applied to the synthesis of a DNA aptamer-grafted photo-responsive hyperbranched polymer, which can self-assemble into nanoparticles and possess numerous hydrophobic inner cavities capable of encapsulating small-molecule therapeutics (Figure 4). ${ }^{76}$ The hyperbranched polymer was synthesized by self-condensing vinyl polymerization of 2-(2bromoisobutyryloxy) ethyl acrylate and oligo(ethylene glycol) acrylate. After polymerization, additional bromine functions were brought in the structure by reacting pendant $\mathrm{OH}$ groups with bromopropionyl chloride. Afterwards, all bromine sites were substituted into reactive azides and reacted with a DCBO-modified aptamer in DMSO. Other examples of SPAAC-mediated aptamer-polymer conjugation were reported. Thurecht and coworkers designed a polymeric nanomedicine platform containing a DMCO-modified DNA aptamer to target triple-negative breast cancer (TNBC) tumors. ${ }^{77}$ 
First, an azido-functionalized hyperbranched polymer was synthesized by reversible addition-fragmentation chain-transfer (RAFT) copolymerization of oligo(ethylene glycol methacrylate), ethylene glycol dimethacrylate and a methacrylamide bearing a near-infrared fluorophore label, in the presence of an azido-functional chain transfer agent. A portion of the azide moieties of the branched copolymer were then modified with DBCO-amine. The amine groups were subsequently functionalized with desferrioxamine isothiocyanate. Finally, a DBCO-functionalized DNA aptamer with high affinity for vascular endothelial growth factor (VEGF)-A (RNV66-DBCO), which was synthesized by phosphoramidite chemistry, was incubated overnight with the azido functionalized polymer to afford the final conjugate. ${ }^{77}$ Very recently, Wang, Tan and coworkers also used DCBO-based SPAAC to prepare aptamer-modified polymeric prodrugs. ${ }^{78}$ In this case, azido-functional copolymers containing both oligo(ethylene glycol) and drug-carrying side chains were synthesized by RAFT polymerization.
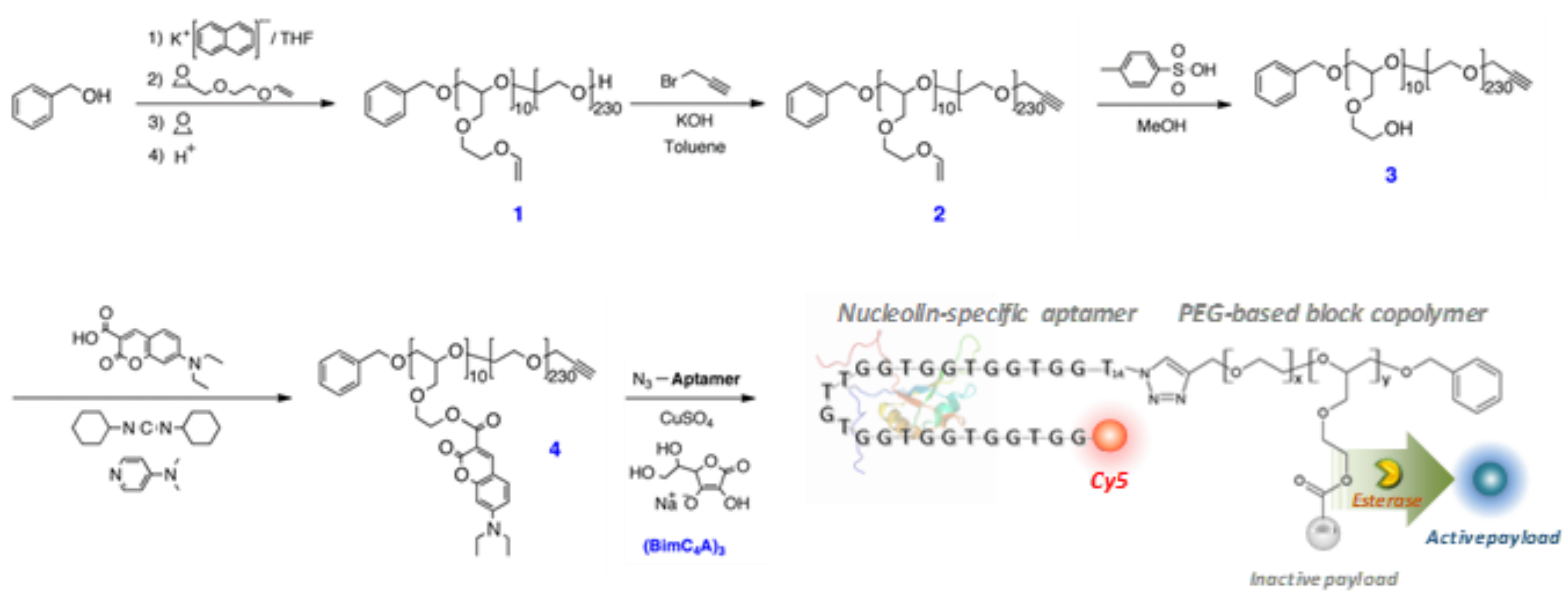

Figure 3. APC synthesis by copper-catalyzed azide-alkyne cycloaddition. In this example, an azido-functional AS1411 aptamer is reacted with an $\omega$-alkyne-functionalized polyether block copolymer (4). The first steps show the different stages of polymer synthesis and modification. Adapted with permission from reference 73. Copyright ACS 2014. 

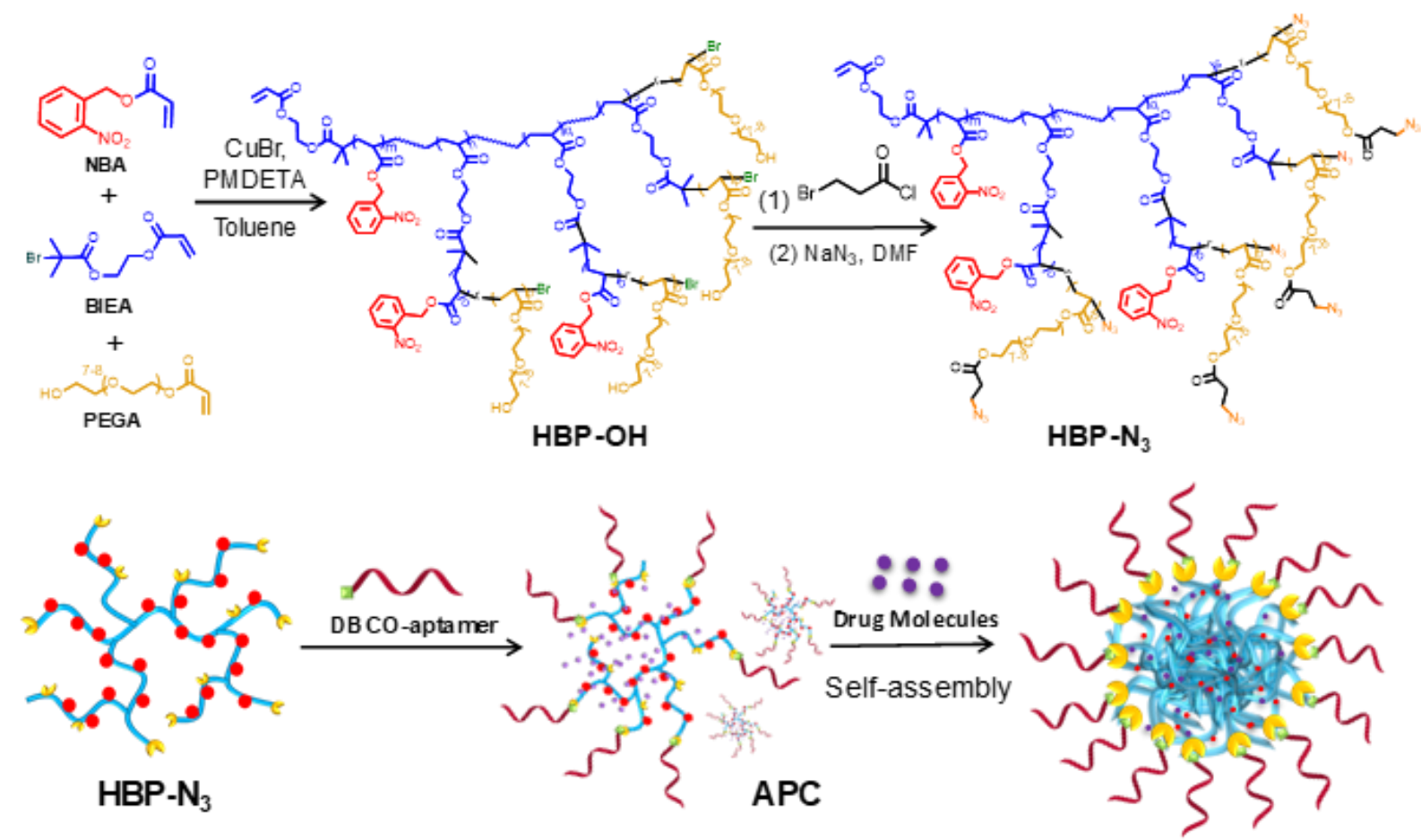

Figure 4. Conjugation of an azido-functionalized hyperbranched polymer with a dibenzocyclooctyne-modified aptamer by strain-promoted metal-free click chemistry. The hydroxy-functional hyperbranched polymer $(\mathrm{HBP}-\mathrm{OH})$ is first obtained by copolymerization 2(2-bromoisobutyryloxy) ethyl acrylate (BIEA), oligo(ethylene glycol) acrylate (PEGA) and ortho-nitrobenzyl acrylate (NBA). The $\mathrm{OH}$ group of the polymer are then esterified with 3bromopropionyl chloride to afford brominated macromolecules. The bromine moieties are then transformed into azide functions by nucleophilic substitution in the presence of sodium azide. The resulting azido-functional hyperbranched polymer $\left(\mathrm{HBP}-\mathrm{N}_{3}\right)$ is then reacted with the DBCO-aptamer. The resulting APC self-assembles into spherical nanoparticles allowing drug encapsulation. Adapted with permission from reference 76. Copyright Wiley VCH 201

\subsubsection{Polymerization of aptamer-based macromonomers}

As mentioned above, the polymerization of macromonomers is a straightforward bioconjugation strategy. ${ }^{7}$ To our knowledge, only one example of this type has been reported so far in the literature by Zhang, Tan and coworkers. ${ }^{79}$ In this work, methacrylamide- 
functionalized aptamers (for example Sgc8) were prepared by solid-phase phosphoramidite chemistry followed by the coupling of the methacrylamide moiety. The formed macromonomers were afterwards copolymerized with acrylamide and dye-containing comonomer in aqueous solution using ammonium persulfate as a radical initiator. As a consequence, the formed water-soluble graft copolymers contain multiple aptamer side-chains.

\subsection{Solid-phase strategies}

\subsubsection{Solid-phase amidation}

The synthesis of amides is typically achieved by the reaction of an activated carboxylic acid with an amine in liquid media, as described in section 2.1 of this review. However, this reaction can also be performed on a solid-support, on which one of the reactants is immobilized. Nucleic acids aptamers are synthesized by solid-phase phosphoramidite chemistry, on a controlled pore glass or crosslinked polystyrene solid support. ${ }^{80,81}$ After synthesis, they are cleaved from the support and purified. However, prior to cleavage, they can be kept on the support for polymer conjugation or other chemical modifications. ${ }^{82,83}$ The conjugates can then easily be separated from other reactants by filtering-off the solid-support. Using such a solid-phase procedure, a resin-bound 5'-amino-functional aptamer can be reacted with a polymer bearing an activated carboxylic acid group, thus resulting in amide formation. For example, Vebert-Nardin and coworkers reported the solid-phase conjugation of an amine-terminated 37-mer aptamer (IgE) with a poly(2-alkyl-2-oxazoline) containing carboxylic acid side-chains. ${ }^{84}$ Before coupling, the carboxylic acid groups of the polymer were activated by $\mathrm{N}, \mathrm{N}^{\prime}$-diisopropylcarbodiimide to react with the amino group of the aptamer. The resulting amphiphilic polymer-aptamer conjugates were then cleaved from the solid-support and were used as droplet stabilizers in oil-in-water and water-in-oil emulsions. A comparable solid-phase conjugation approach was recently 
utilized by Gianneschi and coworkers. ${ }^{85}$ In this case, the synthesis was accomplished by conjugating a support-bound amino-modified thrombin-binding DNA aptamers to a carboxyterminated polymer of ( $\mathrm{N}$-benzyl)-5- norbornene-exo-2,3-dicarboximide. As described above, the aptamer was first synthesized by automated phosphoramidite chemistry on a controlled pore glass support and its chain-end was modified with a commercial amino-modifier. Afterwards, the coupling reaction was carried out in presence of the HATU activating reagent. Finally, the formed polymer-aptamer conjugate was cleaved from the solid support.

\subsubsection{Phosphoramidite Polymer Chemistry}

Solid-phase phosphoramidite chemistry is not restricted to nucleic acids. Indeed, a wide variety of non-biological phosphoramidite monomers can also be used in this approach. ${ }^{86,87}$ In general, such monomers are mainly used to include synthetic moieties in nucleic acids. However, it has been recently demonstrated that this chemistry can also be used to prepare sequence-defined synthetic polymers. ${ }^{88-90}$ Thus, iterative phosphoramidite chemistry is not only the gold standard method for DNA synthesis but also an interesting polymer chemistry tool. ${ }^{91}$ Since this method can be applied to both phosphoramidite nucleosides and non-natural phosphoramidite monomers, it is a very convenient platform for the synthesis of bio-hybrid macromolecules containing both synthetic and DNA segments. ${ }^{92,} 93$ In a recent work of our group, phosphoramidite synthesis was investigated for the first time as a straightforward method for the synthesis of APCs. ${ }^{94}$ Uniform conjugates combining a DNA aptamer segment (either antiMUC1- or ATP-aptamer) and a synthetic polymer segment were successfully synthesized by using both natural and non-natural monomers (e.g. alkyl- and oligo(ethylene glycol)phosphoramidites) (Figure 5). Typically, the nucleic acid segment was synthesized first, followed by the stepwise preparation of the synthetic segment. Hence, to some degree, the present method can be compared to a macroinitiator approach, ${ }^{7}$ even though such a term is not 
perfectly accurate for a multistep-growth synthesis. ${ }^{95}$ Overall, automated phosphoramidite chemistry allows synthesis of complete APCs with an absolute size and monomer sequence control for both the DNA and synthetic segment. For instance, APCs containing synthetic block copolymer segments with perfectly-controlled block sizes were synthesized in this work. Importantly, it was also demonstrated using circular dichroism spectroscopy that polymer conjugation does not prevent aptamer folding.

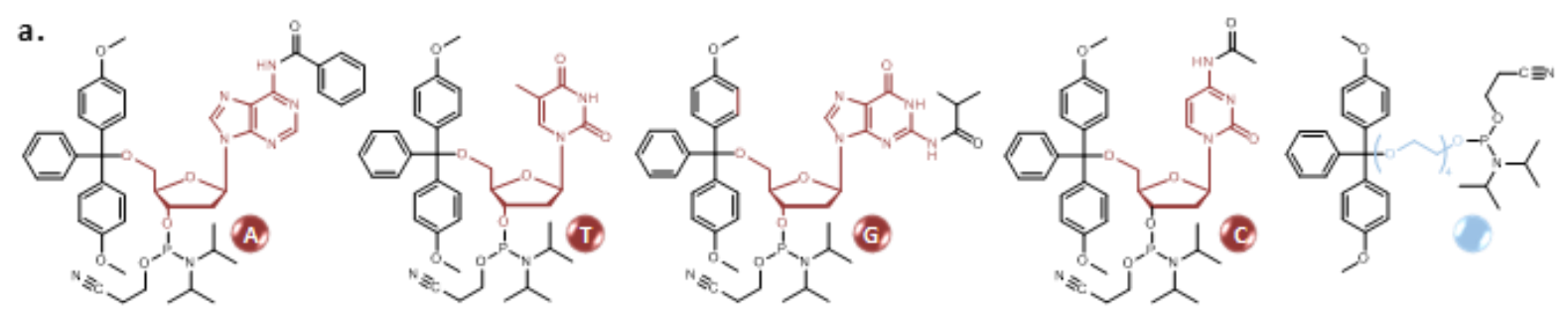

b.

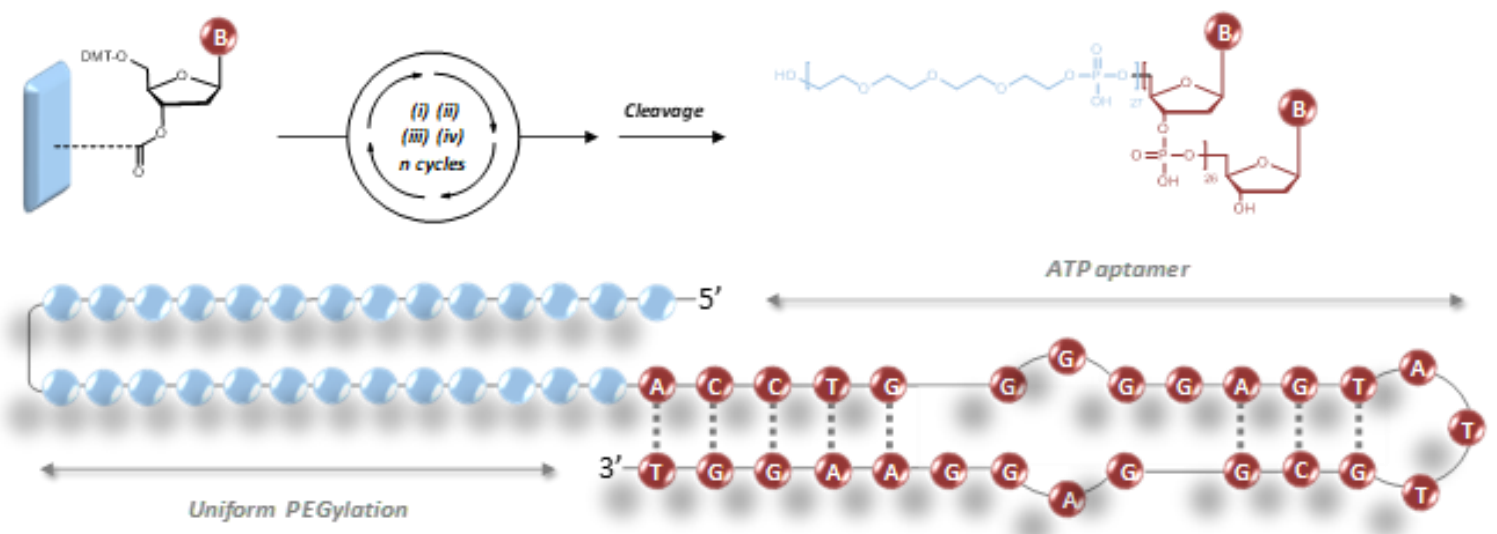

Figure 5. APC preparation of a molecularly-uniform APC by phosphoramidite polymer chemistry. ${ }^{94}$ (a) molecular structure of the phosphoramidite monomers. Red monomers are natural nucleotides, whereas the blue one is an unnatural building block. (b) Stepwise solidphase phosphoramidite chemistry leading to the synthesis of a uniform APC combining a PEGylated segment to the ATP aptamer.

\section{Non-covalent conjugations}

While covalent strategies are predominantly reported for aptamer conjugation to polymers, non-covalent interactions have also been explored to prepare APCs with unique properties. For instance, the electrostatic complexation of molecules of opposite 
charge has been investigated as an alternative bioconjugation method for the development of aptamer-targeted drug and gene delivery nanosystems for cancer therapy. In particular, it has been used to complex electrostatically the negatively charged phosphate backbone of aptamers with cationic polymeric nanoparticles loaded with drugs or therapeutic genes. Such a strategy has mainly been used to complex polyethylenimine (PEI), which is the most studied synthetic cationic polymer for gene delivery, with negatively charged therapeutic nucleic acids in the form of plasmid DNA (pDNA) and/or in the form of small interfering RNA (siRNA), short hairpin RNA (shRNA) or micro RNA (miRNA). The resulting complexes, commonly known as polyplexes, have played an important role in aptamer targeted non-viral gene delivery, as they can protect nucleic acids from enzymatic degradation and enable cargo release selectively to tumor sites. For instance, in order to fabricate an efficient cell/ tissuespecific delivery system which exclusively targets and affects human anaplastic large cell lymphoma (ALCL), Zu and co-workers prepared nanocomplexes by incorporating both CD30 aptamer and ALK siRNA into PEI polymer carriers. ${ }^{96}$ First, a nano-sized core was formed by crosslinking branched PEI with sodium citrate. Then, the synthetic CD30 aptamers and ALK siRNAs were complexed with the PEI-citrate core, to form a complex with a diameter of about $140 \mathrm{~nm}$. Zeta potential measurements confirmed that the initially positively-charged PEI-citrate nanocore became negatively-charged after oligonucleotides complexation. Using a comparable strategy, polyplexes for targeted gene delivery were prepared by Abnous, Ramezani and coworkers. ${ }^{97}$ For this purpose, the sgc-8c aptamer was non-covalently conjugated to pre-formed polyplexes consisting of pDNA and PEI. Conjugation of the aptamer to PEI increased the size of the polyplexes and decreased their zeta potential. Moreover, dynamic light scattering and transmission electron microscopy measurements indicated that aptamer-containing polyplexes of 
varying sizes (160-275 nm) could be prepared by using different pDNA:PEI:aptamer ratios. In addition, Sasaki and coworkers managed to coat electrostatically cationic pDNA/PEI complexes with the MUC1 aptamer. ${ }^{98}$ The resulting tumor-targeted gene delivery vectors showed high gene expressions of the plasmid-borne luciferase gene in A549 cells expressing MUC1 regularly under in vitro and in vivo conditions.

Polyelectrolyte complexes of cationic polysaccharides such as chitosan with anionic polysaccharides like dextran sulfate, carboxymethyl cellulose and alginates have been also studied as aptamer-targeted delivery systems, because of their excellent biocompatibility and surface charge properties. For example, aiming to enhance the permeability and retention time of tumor suppressive miRNAs, Atyabi and coworkers developed complexes prepared from chitosan and miR-14 conjugated thiolated dextran. ${ }^{99}$ The size of the resulting complexes was between $40-270 \mathrm{~nm}$, and the zeta potential varied depending on the TD-miR/chitosan molar ratio. Then, in order to achieve targeted intracellular drug and gene delivery the anti-nucleolin aptamer AS1411 was non-covalently conjugated to the surface of the complexes through electrostatic interactions between the negatively-charged phosphate groups of the aptamer and the positively-charged amine groups of chitosan on the surface of the complexes. The outcomes of cellular studies indicated that the resulting non-covalent APCs were remarkably efficient for delivery of cytotoxic chemotherapeutics because of their double targeted nature that reduces drug dose and side effects on normal cells. Abnous, Taghdisi and coworkers reported the synthesis of epirubicin-loaded PLGA nanoparticles with a chitosan outer-layer. ${ }^{100}$ The epirubicin-loaded PLGA nanoparticles were first prepared by an emulsion and solvent evaporation technique. The surface of the resulting nanoparticles was then modified with chitosan by electrostatic adsorption. This led to a 
net positive surface charge, and thus allowed electrostatic conjugation of MUC1 aptamer.

Apart from gene delivery carriers, non-covalent aptamer conjugation has also been explored for the preparation of bio-sensors. For instance, a molecular beacon aptamer, containing an ATP specific aptamer, a fluorophore and a quencher, was electrostaticallyconjugated to cationic poly(fluorene-phenylene) and was studied as a potential ATP assay. ${ }^{101}$ In the absence of ATP, the conjugated polymer form an open-chain electrostatic complex with the aptamer, thus leading to fluorescence resonance energy transfer (from the conducting polymer to the fluorophore in the molecular beacon aptamer). In the presence of ATP, the hairpin-shaped molecular beacon aptamer captures ATP by forming a G-quadruplex. The ATP-specific folding brings the fluorophore and the quencher in contact, thus resulting in quenched photoluminescence.

Overall, non-covalent conjugation allows facile and rapid preparation of aptamerpolymer aggregates. However, when using a simple supramolecular concept such as the association of oppositely-charged polyelectrolytes, this strategy is less controlled than covalent conjugation. Indeed, it only gives a rough control over molecular and morphological parameters such as polymer/aptamer ratio, aggregation number and functionality. However, more precise non-covalent conjugations could be envisioned using discrete synthetic segments containing supramolecular motifs (e.g. hydrogen bonding moieties). This could lead to the formation of defined tertiary structures, involving both nucleic acid and synthetic segments. Yet, such opportunities have not been explored to date.

\section{Conclusions}

As shown in this review, a certain number of APCs have been described in the literature to date. Many of them have been synthesized using highly-established bioconjugation 
tools such as NHS-activated amidation or thiol-maleimide chemistry. Although efficient and widespread, such chemistries are not necessarily leading to well-defined conjugates. They often require large excess of one reactant (i.e. aptamer or synthetic polymer) to maximize the conjugate yield and, when performed in solution, they require stringent purifications to remove unreacted macromolecular reagents. Thus, more thoughtful polymer chemistry approaches have been reported, for example employing click chemistry tools such as CuAAC, SPAAC. Overall, these modern conjugation approaches enable synthesis of APCs with controlled molecular weight, polydispersity and functionality. Furthermore, using solid-phase automated phosphoramidite chemistry, it is even possible to prepare uniform APCs, in which both the synthetic and biological segments are perfectly sequence-defined. In this case, the use of a solid-phase support also greatly facilitates purification and isolation.

In terms of analytical characterization, many of the reported APCs have only been partly described. For instance, the influence of the attachment (covalent or non-covalent) of synthetic polymer segments on the folding and conformation of DNA and RNA aptamers is not systematically investigated. In-depth analytical studies by circular dichroism, UV spectroscopy and X-ray crystallography would be beneficial for the field. Furthermore, most of the applications explored for APCs deal with biomedical or biotechnological applications, such a drug delivery, gene therapy and tissue engineering. However, the conjugation of synthetic polymers and nucleic acid aptamers can also lead to interesting new types of smart materials. ${ }^{102}$ For instance, the well-known ATP-binding DNA aptamer opens up very exciting possibilities in applied materials science. ${ }^{103}$ Thus, APCs may also be used in the future as chemically-responsive or bio-responsive components in dynamic macromolecular networks. This also opens up very interesting perspectives for macromolecular systems chemistry. ${ }^{104}$ 


\section{Acknowledgements}

M.N. thanks the Bodossaki Foundation (Athens, Greece) for research funding for the period 2018-2021. J.F-L. thanks the CNRS and the Institut Thématique Interdisciplinaire "Chimie des Systèmes Complexes" (ITI-CSC) for financial support. The PhD position of C.L. is supported by the ITI-CSC. 


\section{References}

1. A. D. Ellington and J. W. Szostak, Nature, 1990, 346, 818-822.

2. C. Tuerk and L. Gold, Science, 1990, 249, 505-510.

3. T. Hermann and D. J. Patel, Science, 2000, 287, 820.

4. M. Famulok, J. S. Hartig and G. Mayer, Chem. Rev., 2007, 107, 3715-3743.

5. A. D. Keefe, S. Pai and A. Ellington, Nat. Rev. Drug Discovery, 2010, 9, 537-550.

6. A. B. Iliuk, L. Hu and W. A. Tao, Anal. Chem., 2011, 83, 4440-4452.

7. J.-F. Lutz and H. G. Börner, Prog. Polym. Sci., 2008, 33, 1-39.

8. C. Chen, D. Y. W. Ng and T. Weil, Prog. Polym. Sci., 2020, 105, 101241.

9. K. Knop, R. Hoogenboom, D. Fischer and U. S. Schubert, Angew. Chem., Int. Ed., 2010, 49, 6288-6308.

10. E. W. M. Ng, D. T. Shima, P. Calias, E. T. Cunningham, D. R. Guyer and A. P. Adamis, Nat. Rev. Drug Discovery, 2006, 5, 123-132.

11. J. Cheng, B. A. Teply, I. Sherifi, J. Sung, G. Luther, F. X. Gu, E. Levy-Nissenbaum, A. F. Radovic-Moreno, R. Langer and O. C. Farokhzad, Biomaterials, 2007, 28, 869876.

12. H. Yang, H. Liu, H. Kang and W. Tan, J. Am. Chem. Soc., 2008, 130, 6320-6321.

13. I. Willner and M. Zayats, Angew. Chem., Int. Ed., 2007, 46, 6408-6418.

14. W. Tan, H. Wang, Y. Chen, X. Zhang, H. Zhu, C. Yang, R. Yang and C. Liu, Trends Biotechnol., 2011, 29, 634-640.

15. Q. Zhao, M. Wu, X. Chris Le and X.-F. Li, TrAC, Trends Anal. Chem., 2012, 41, 4657.

16. L. C. Bock, L. C. Griffin, J. A. Latham, E. H. Vermaas and J. J. Toole, Nature, 1992, 355, 564-566.

17. M. Marušič, R. N. Veedu, J. Wengel and J. Plavec, Nucleic Acids Res., 2013, 41, 9524-9536.

18. C. S. Ferreira, C. S. Matthews and S. Missailidis, Tumour Biol., 2006, 27, 289-301.

19. P. J. Bates, D. A. Laber, D. M. Miller, S. D. Thomas and J. O. Trent, Exp. Mol. Pathol., 2009, 86, 151-164.

20. D. E. Huizenga and J. W. Szostak, Biochemistry, 1995, 34, 656-665.

21. Z. Bognár and R. E. Gyurcsányi, Int. J. Mol. Sci., 2020, 21. 
22. I. P. M. Lau, E. K. S. Ngan, J. F. C. Loo, Y. K. Suen, H. P. Ho and S. K. Kong, Biochem. Biophys. Res. Commun., 2010, 395, 560-564.

23. D. Shangguan, Z. Tang, P. Mallikaratchy, Z. Xiao and W. Tan, ChemBioChem, 2007, 8, 603-606.

24. A. Prodeus, A. Abdul-Wahid, N. W. Fischer, E. H. Huang, M. Cydzik and J. Gariépy, Mol. Ther. Nucleic Acids, 2015, 4, e237.

25. T. Mori, A. Oguro, T. Ohtsu and Y. Nakamura, Nucleic Acids Res., 2004, 32, 61206128.

26. S. E. Lupold, B. J. Hicke, Y. Lin and D. S. Coffey, Cancer Res., 2002, 62, 4029.

27. B. J. Hicke, C. Marion, Y.-F. Chang, T. Gould, C. K. Lynott, D. Parma, P. G. Schmidt and S. Warren, J. Biol. Chem., 2001, 276, 48644-48654.

28. S. Shigdar, J. Lin, Y. Yu, M. Pastuovic, M. Wei and W. Duan, Cancer Sci., 2011, 102, 991-998.

29. B. J. Hicke, A. W. Stephens, T. Gould, Y. F. Chang, C. K. Lynott, J. Heil, S. Borkowski, C. S. Hilger, G. Cook, S. Warren and P. G. Schmidt, J. Nucl. Med., 2006, 47, 668-678.

30. L. Tan, K. G. Neoh, E. T. Kang, W. S. Choe and X. Su, Macromol. Biosci., 2011, 11, 1331-1335.

31. C. S. Ferreira, K. Papamichael, G. Guilbault, T. Schwarzacher, J. Gariepy and S. Missailidis, Anal Bioanal Chem, 2008, 390, 1039-1050.

32. O. C. Farokhzad, J. Cheng, B. A. Teply, I. Sherifi, S. Jon, P. W. Kantoff, J. P. Richie and R. Langer, Proc. Natl. Acad. Sci. U.S.A. ～, 2006, 103, 6315-6320.

33. S. Dhar, F. X. Gu, R. Langer, O. C. Farokhzad and S. J. Lippard, Proc. Natl. Acad. Sci. U.S.A. , 2008, 105, 17356-17361.

34. L. Zhang, J. M. Chan, F. X. Gu, J.-W. Rhee, A. Z. Wang, A. F. Radovic-Moreno, F. Alexis, R. Langer and O. C. Farokhzad, ACS nano, 2008, 2, 1696-1702.

35. J. Guo, X. Gao, L. Su, H. Xia, G. Gu, Z. Pang, X. Jiang, L. Yao, J. Chen and H. Chen, Biomaterials, 2011, 32, 8010-8020.

36. C. Yu, Y. Hu, J. Duan, W. Yuan, C. Wang, H. Xu and X.-D. Yang, PLOS ONE, 2011, 6, e24077.

37. A. Aravind, R. Nair, S. Raveendran, S. Veeranarayanan, Y. Nagaoka, T. Fukuda, T. Hasumura, H. Morimoto, Y. Yoshida, T. Maekawa and D. Sakthi Kumar, J. Magn. Magn. Mater., 2013, 344, 116-123.

38. M. Das, W. Duan and S. K. Sahoo, Nanomedicine, 2015, 11, 379-389. 
39. M. Ni, M. Xiong, X. Zhang, G. Cai, H. Chen, Q. Zeng and Z. Yu, Int. J. Nanomed., 2015, 10, 2537-2554.

40. J. Mosafer, M. Teymouri, K. Abnous, M. Tafaghodi and M. Ramezani, Mater. Sci. Eng., C, 2017, 72, 123-133.

41. S. Vandghanooni, M. Eskandani, J. Barar and Y. Omidi, Nanomedicine (Lond), 2018, 13, 2729-2758.

42. T. Duan, Z. Xu, F. Sun, Y. Wang, J. Zhang, C. Luo and M. Wang, Biomed. Pharmacother., 2019, 117, 109121.

43. S. Chakraborty, Z. Y. Dlie, S. Chakraborty, S. Roy, B. Mukherjee, S. E. Besra, S. Dewanjee, A. Mukherjee, P. K. Ojha, V. Kumar and R. Sen, Mol. Ther. Nucleic Acids, 2020, 20, 34-49.

44. K. X. Tan, S. Y. Lau and M. K. Danquah, Biomed. Pharmacother., 2018, 101, 9961002.

45. R. Tong, L. Yala, T. M. Fan and J. Cheng, Biomaterials, 2010, 31, 3043-3053.

46. S. V. Lale, A. R. G, A. Aravind, D. S. Kumar and V. Koul, Biomacromolecules, 2014, 15, 1737-1752.

47. Z. Luo, Z. Yan, K. Jin, Q. Pang, T. Jiang, H. Lu, X. Liu, Z. Pang, L. Yu and X. Jiang, J. Colloid Interface Sci., 2017, 490, 783-796.

48. X. Li, Y. Yu, Q. Ji and L. Qiu, Nanomedicine, 2015, 11, 175-184.

49. J. Zhang, R. Chen, X. Fang, F. Chen, Y. Wang and M. Chen, Nano Res., 2015, 8, 201218.

50. S. Yang, Z. Ren, M. Chen, Y. Wang, B. You, W. Chen, C. Qu, Y. Liu and X. Zhang, Mol. Pharm., 2018, 15, 314-325.

51. S. Engelberg, E. Netzer, Y. G. Assaraf and Y. D. Livney, Cell Death Dis., 2019, 10, 702.

52. M. Alibolandi, M. Ramezani, K. Abnous and F. Hadizadeh, J. Pharm. Sci., 2016, 105, 1741-1750.

53. P. Ramezani, K. Abnous, S. M. Taghdisi, M. Zahiri, M. Ramezani and M. Alibolandi, Colloids Surf., B, 2020, 193, 111135.

54. J. Zhou, B. Soontornworajit and Y. Wang, Biomacromolecules, 2010, 11, 2087-2093.

55. S. Ayatollahi, Z. Salmasi, M. Hashemi, S. Askarian, R. K. Oskuee, K. Abnous and M. Ramezani, Int. J. Biochem. Cell Biol., 2017, 92, 210-217.

56. W. Xu, I. A. Siddiqui, M. Nihal, S. Pilla, K. Rosenthal, H. Mukhtar and S. Gong, Biomaterials, 2013, 34, 5244-5253.

57. C. E. Hoyle and C. N. Bowman, Angew. Chem., Int. Ed., 2010, 49, 1540-1573. 
58. A. B. Lowe, C. E. Hoyle and C. N. Bowman, J. Mater. Chem., 2010, 20, 4745-4750.

59. D. P. Nair, M. Podgórski, S. Chatani, T. Gong, W. Xi, C. R. Fenoli and C. N. Bowman, Chem. Mater., 2014, 26, 724-744.

60. C. Da Pieve, P. Williams, D. M. Haddleton, R. M. J. Palmer and S. Missailidis, Bioconjugate Chem., 2010, 21, 169-174.

61. J.-F. Lutz, J. Polym. Sci., Part A: Polym. Chem., 2008, 46, 3459-3470.

62. C. Da Pieve, E. Blackshaw, S. Missailidis and A. C. Perkins, Bioconjugate Chem., 2012, 23, 1377-1381.

63. X. Huang, J. Wan, D. Leng, Y. Zhang and S. Yang, Exp. Ther. Med., 2020, 19, 192204.

64. M. Alibolandi, S. M. Taghdisi, P. Ramezani, F. Hosseini Shamili, S. A. Farzad, K. Abnous and M. Ramezani, Int. J. Pharm., 2017, 519, 352-364.

65. A. Barzegar Behrooz, F. Nabavizadeh, J. Adiban, M. Shafiee Ardestani, R. Vahabpour, M. R. Aghasadeghi and H. Sohanaki, Clin. Exp. Pharmacol. Physiol., 2017, 44, 41-51.

66. H. Chen, J. Tian, D. Liu, W. He and Z. Guo, J. Mater. Chem. B, 2017, 5, 972-979.

67. G. Xu, X. Yu, J. Zhang, Y. Sheng, G. Liu, W. Tao and L. Mei, Int. J. Nanomed., 2016, 11, 2953-2965.

68. H. Lee, S. M. Dellatore, W. M. Miller and P. B. Messersmith, Science, 2007, 318, 426.

69. N. Kong, M. Deng, X.-N. Sun, Y.-D. Chen and X.-B. Sui, Front. Pharmacol., 2018, 9.

70. Y. Sugawara, T. Tamaki and T. Yamaghchi, Polymer, 2015, 62, 86-93.

71. J.-F. Lutz, Angew. Chem., Int. Ed., 2007, 46, 1018-1025.

72. J.-F. Lutz and Z. Zarafshani, Adv. Drug Delivery Rev., 2008, 60, 958-970.

73. S. S. Oh, B. F. Lee, F. A. Leibfarth, M. Eisenstein, M. J. Robb, N. A. Lynd, C. J. Hawker and H. T. Soh, J. Am. Chem. Soc., 2014, 136, 15010-15015.

74. N. J. Agard, J. A. Prescher and C. R. Bertozzi, J. Am. Chem. Soc., 2004, 126, 1504615047.

75. J.-F. Lutz, Angew. Chem., Int. Ed., 2008, 47, 2182-2184.

76. L. Yang, H. Sun, Y. Liu, W. Hou, Y. Yang, R. Cai, C. Cui, P. Zhang, X. Pan, X. Li, L. Li, B. S. Sumerlin and W. Tan, Angew. Chem., Int. Ed., 2018, 57, 17048-17052.

77. N. L. Fletcher, Z. H. Houston, J. D. Simpson, R. N. Veedu and K. J. Thurecht, Chem. Commun., 2018, 54, 11538-11541. 
78. Z. Deng, Q. Yang, Y. Peng, J. He, S. Xu, D. Wang, T. Peng, R. Wang, X.-Q. Wang and W. Tan, Bioconjugate Chem., 2020, 31, 37-42.

79. L. Yang, L. Meng, X. Zhang, Y. Chen, G. Zhu, H. Liu, X. Xiong, K. Sefah and W. Tan, J. Am. Chem. Soc., 2011, 133, 13380-13386.

80. M. H. Caruthers, Science, 1985, 230, 281-285.

81. S. L. Beaucage and R. P. Iyer, Tetrahedron, 1992, 48, 2223-2311.

82. F. E. Alemdaroglu, M. Safak, J. Wang, R. Berger and A. Herrmann, Chem. Commun., 2007, DOI: 10.1039/B615276G, 1358-1359.

83. T. Schnitzler and A. Herrmann, Acc. Chem. Res., 2012, 45, 1419-1430.

84. D. Kedracki, P. Maroni, H. Schlaad and C. Vebert-Nardin, Adv. Funct. Mater., 2014, 24, 1133-1139.

85. A. Roloff, A. S. Carlini, C. E. Callmann and N. C. Gianneschi, J. Am. Chem. Soc., 2017, 139, 16442-16445.

86. N. Appukutti and C. J. Serpell, Polym. Chem., 2018, 9, 2210-2226.

87. M. Vybornyi, Y. Vyborna and R. Häner, Chem. Soc. Rev., 2019, 48, 4347-4360.

88. A. Al Ouahabi, L. Charles and J.-F. Lutz, J. Am. Chem. Soc., 2015, 137, 5629-5635.

89. A. Al Ouahabi, M. Kotera, L. Charles and J.-F. Lutz, ACS Macro Lett., 2015, 4, 10771080.

90. L. Charles and J.-F. Lutz, Acc. Chem. Res., 2021, 54, 1791-1800.

91. J.-F. Lutz, M. Ouchi, D. R. Liu and M. Sawamoto, Science, 2013, 341, 1238149.

92. T. G. W. Edwardson, K. M. M. Carneiro, C. J. Serpell and H. F. Sleiman, Angew. Chem., Int. Ed., 2014, 53, 4567-4571.

93. T. Mondal, M. Nerantzaki, K. Flesch, C. Loth, M. Maaloum, Y. Cong, S. S. Sheiko and J.-F. Lutz, Macromolecules, 2021, 54, 3423-3429.

94. C. Loth, L. Charles, J.-F. Lutz and M. Nerantzaki, ACS Macro Letters, 2021, 10, 481485.

95. J.-F. Lutz, J.-M. Lehn, E. W. Meijer and K. Matyjaszewski, Nat. Rev. Mater., 2016, 1, 16024.

96. N. Zhao, H. G. Bagaria, M. S. Wong and Y. Zu, J. Nanobiotechnol., 2011, 9, 2-2.

97. N. Shahidi-Hamedani, W. T. Shier, F. Moghadam Ariaee, K. Abnous and M. Ramezani, J. Gene Med., 2013, 15, 261-269.

98. T. Kurosaki, N. Higuchi, S. Kawakami, Y. Higuchi, T. Nakamura, T. Kitahara, M. Hashida and H. Sasaki, Gene, 2012, 491, 205-209. 
99. F. S. M. Tekie, M. Soleimani, A. Zakerian, M. Dinarvand, M. Amini, R. Dinarvand, E. Arefian and F. Atyabi, Carbohydr. Polym., 2018, 201, 131-140.

100. S. Taghavi, M. Ramezani, M. Alibolandi, K. Abnous and S. M. Taghdisi, Cancer Lett., 2017, 400, 1-8.

101. J. E. Jeong and H. Y. Woo, Polym. Chem., 2017, 8, 6329-6334.

102. E. Mastronardi, A. Foster, X. Zhang and M. C. DeRosa, Sensors (Basel, Switzerland), 2014, 14, 3156-3171.

103. J. Deng and A. Walther, Adv. Mater., 2020, 32, 2002629.

104. J.-F. Lutz, ACS Macro Lett., 2020, 9, 185-189. 